\title{
Laryngeal and Ocular Granulation Tissue Formation in Two Punjabi Children: LOGIC Syndrome
}

\author{
J. R. AINSWORTH* , A. FIONA SPENCER*, J. DUDGEON*, N. K. GEDDES $\dagger$, \\ W. R. LEE $\ddagger$ \\ Glasgow
}

\begin{abstract}
Summary
Two unrelated children, both born in the United Kingdom of Punjabi Muslim parents, developed within months of birth, nodules in the larynx, conjunctiva and nailbeds. Currently the younger female child, aged five, is developing skin papules while the elder boy, now aged 15, is debilitated by oral, oesophageal and tracheal granulation tissue formation. Numerous biopsies of the affected tissues have revealed subepithelial granulation tissue fromation of unknown aetiology. Extensive haematological, biochemical and bacteriological investigations were uninformative as to the cause. Treatment with steroids, dapsone, antituberculous drugs, and local excision failed to control the disease.

No description of this condition exists in the international medical literature available to us.
\end{abstract}

Chronic scarring conjunctival disease is very rare in children of the developed world, and usually represents only one aspect of a recognised systemic condition such as epidermolysis bullosa ${ }^{1}$ or cicatricial pemphigoid. ${ }^{2}$ In this report we describe the clinical and laboratory features in a multisystem disorder that affected two children from the first months of life. The syndrome was characterised by the formation of granulation tissue in the dermis and submucosa of several structures including larynx, skin, nailbed and conjunctiva, with subsequent ulceration. Progression of the disease has been documented through the first fifteen years of life.

\section{Case 1}

Clinical features

A three month old Muslim boy presented with a history of a weak cry since birth. He was born in 1976 in Scotland of Punjabi parents who were second cousins.

Examination in the ENT clinic at this time revealed no abnormality. He reattended at six months of age with bleeding from small collections of granulation tissue under his nails and from mucosal surfaces of the mouth. Examination of the larynx under anaesthetic revealed thickening and ulceration of the anterior two-thirds of the vocal cords. At the age of nine months a fleshy conjunctival mass appeared at the lateral fornix of each eye. Biopsy of the mass demonstrated marked thickening of the submucosa by granulation tissue.

The child suffered a slow but relentless progression of the disease over the subsequent five years (Fig. 1). The larynx was infiltrated

Departments of *Ophthalmology and †ENT, Royal Hospital for Sick Children, and Departments of *Ophthalmology and $\ddagger$ Pathology, Tennent Institute, Western Infirmary, Glasgow.

Correspondence to: Dr J. R. Ainsworth, Tennent Institute of Ophthalmology, Western Infirmary, Glasgow G11 $6 \mathrm{NT}$. 


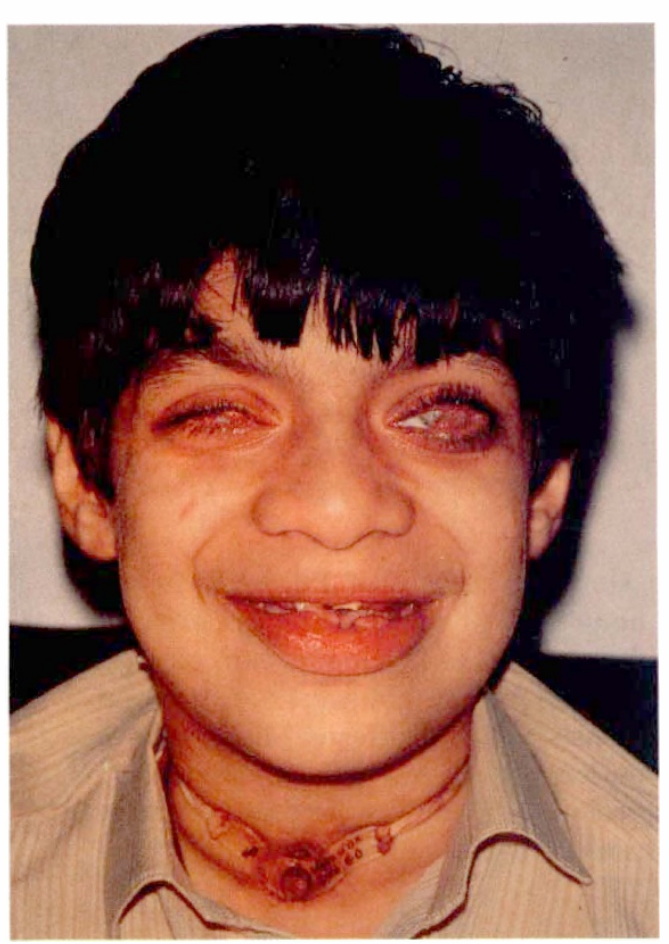

Fig. 1. In case 1 a permanent tracheostomy became necessary by five years of age because of recurrent upper airway obstruction. Conjunctival and gingival granulation tissue is present.

by granulation tissue extending from the vocal cords and obstructing the glottis. A permanent tracheostomy was necessary by his fifth birthday. Granulation tissue and symblepharon formation extended medially from the lateral fornices and, combined with xerosis,

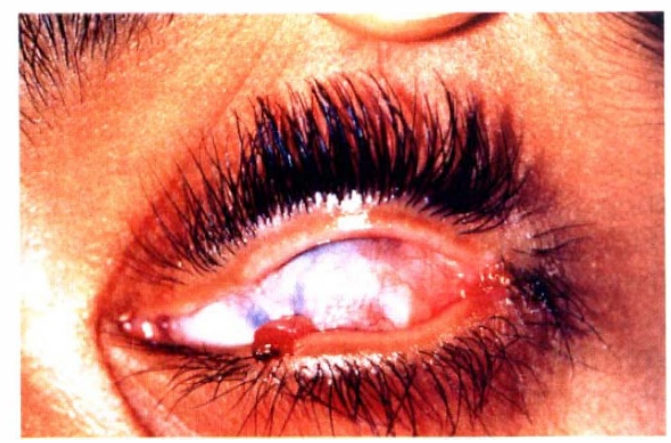

Fig. 2. Case 1 at fourteen years of age. There is symblepharon formation between the inferior eyelid and cornea. Visual acuity in each eye is perception of light due to corneal opacification. eventually resulted in corneal opacification (Fig. 2). In addition exuberant masses of abnormal tissue appeared in the gums with loss of several teeth (Fig. 3).

By his ninth birthday he had developed a distal oesophageal stricture (Fig. 4) due to submucosal thickening and ulceration. Over the last three years the laryngeal disease has spread to involve the epiglottis and trachea. He has spontaneously lost finger and toe nails on several occasions but these have grown back with mild residual distortion of the nailbed. Small ulcers on ear, arm and penis were noted in the first years of life but they resolved during his fourth year: the only other apparent dermatological abnormality was of poor wound healing noted by the mother. No bullae or milia were present at any time to suggest epidermolysis bullosa, and the skin has normal adnexal structures with no hyperkeratosis. Surgical excision and local or systemic steroid therapy did not lead to improvement in any aspect of the disease.

The patient is now fifteen years old and has a visual acuity in each eye of light perception only. He requires regular hospital admissions for supplemental nutrition, modification of tracheostomy and control of the oral granulation. He receives regular blood transfusion to correct anaemia resulting from bleeding of the oesophageal stricture and tracheostomy site.

His parents and a 10 year old brother are unaffected.

\section{Laboratory investigations}

At least twelve biopsies have been taken from

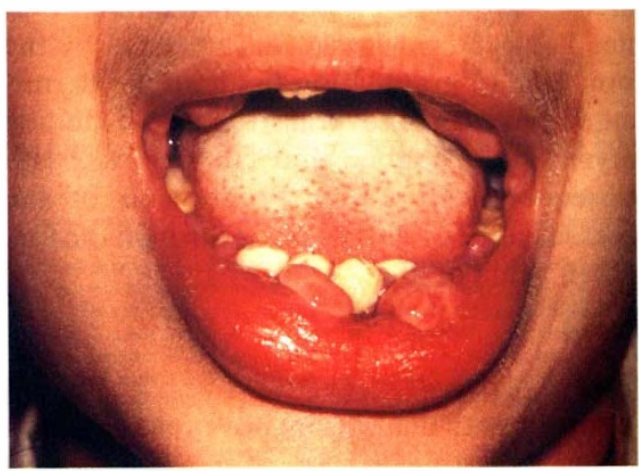

Fig. 3. At nine years of age case 1 has developed large masses of submucosal granulation tissue which is associated with gingival ulceration. Dental enamel is absent giving the teeth a yellow appearance. 


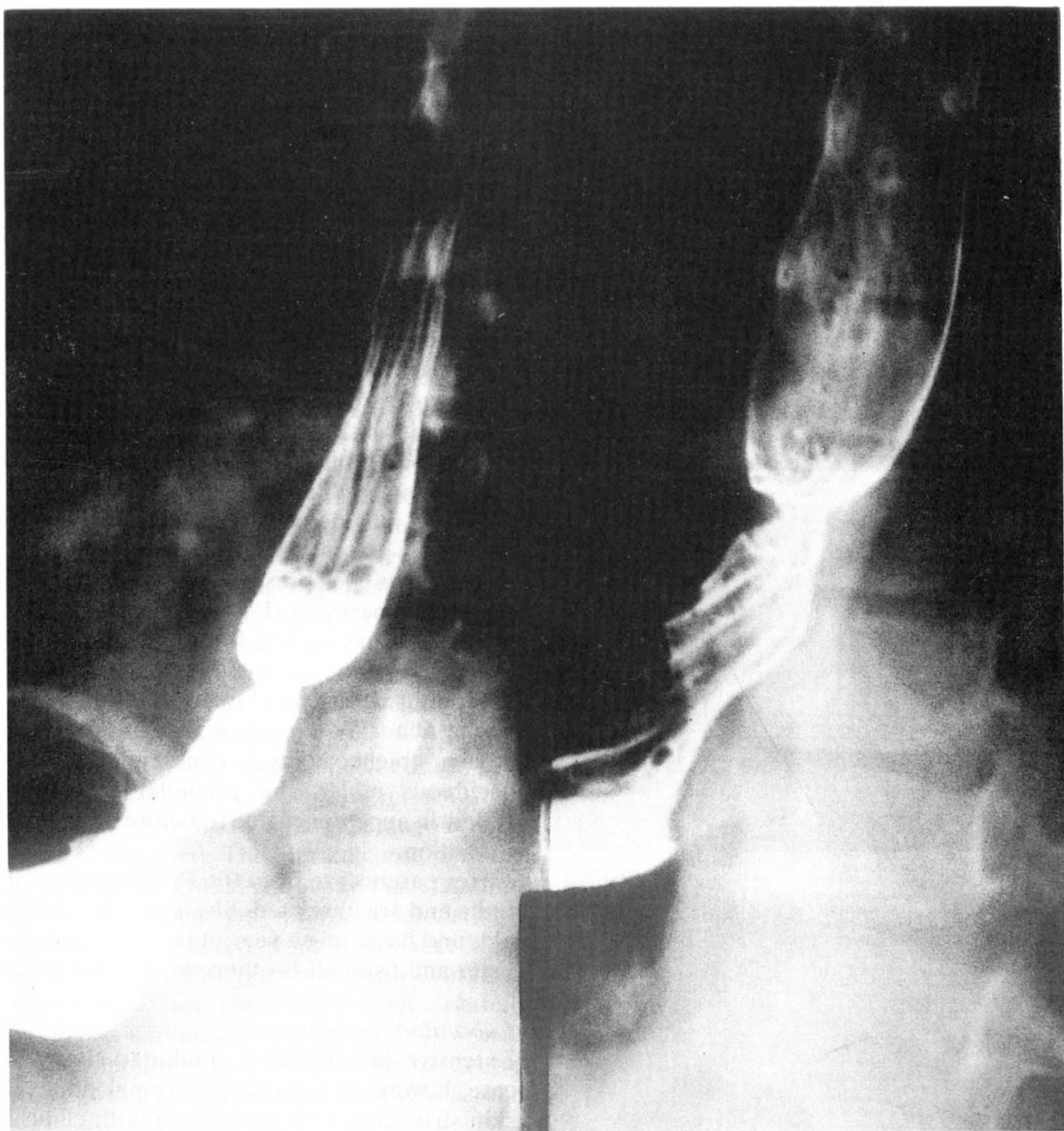

Fig. 4. Barium meal performed when case 1 was nine years old. A stricture is present in the distal oesophagus.

affected areas during the 15 year course of the disease, all of which demonstrated a consistent finding of extensive submucosal granulation tissue on paraffin section (Fig. 5). Immunofluorescence studies and electron microscopy failed to show any changes specific to mechanobullous or immune complex disease. Special stains failed to demonstrate pathogenic organisms. Despite the absence of clinically apparent skin involvement biopsies revealed marked perivascular lymphocytic cuffing with generalised dermal thickening (Fig. 5a). Dental histology revealed near total failure of enamel formation. Rectal and bone marrow biopsy revealed no abnormality of note. Extensive biochemical screening showed no abnormality. A normal karyotype was seen on chromosomal analysis.

\section{Case 2}

\section{Clinical features}

A three month old Muslim girl who had lived all her life in Scotland presented in 1986 with a six week history of a "squeaky cry" and stridor. A mass excised from the right vocal 


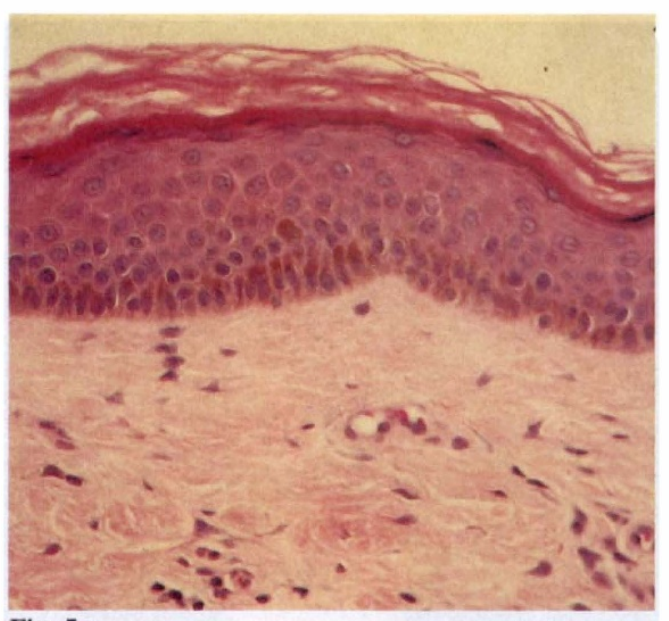

Fig. 5a.

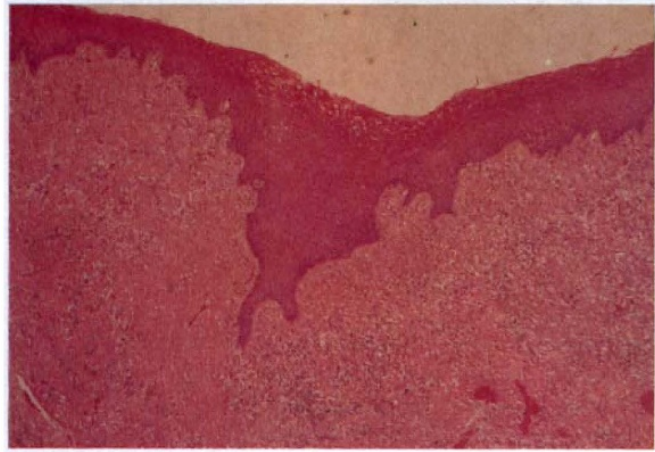

Fig. 5b.

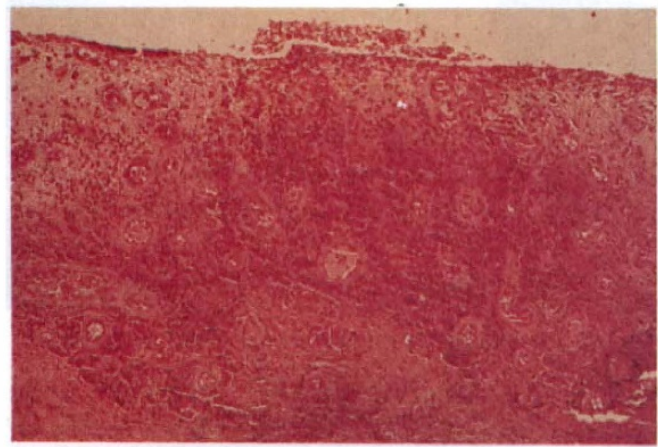

Fig. 5c.

Fig. 5. (a) Histology of apparently unaffected skin in case 1 reveals non-specific perivascular lymphocytic cuffing and a generalised fibrosis of the dermis (Haematoxylin \& Eosin, $\times 400)$.

(b) Clinically affected areas show marked submucosal or dermal granulation tissue formation, as seen on this biopsy of gingiva from case 1 (Haematoxylin and Eosin, $\times 160$ ).

(c) After a period the epithelium overlying the granulation tissue ulcerates, as demonstrated on a conjunctival biopsy from case 2 (Haematoxylin \& Eosin $\times 160$ ). cord. This consisted of granulation tissue without any indication of a specific aetiology. In the ensuing months she developed fleshy conjunctival masses at both lateral canthi with associated symblephara (Fig. 6). Concurrently, the patient developed right, then left corneal epithelial defects that healed slowly with the use of chloramphenicol ointment. The conjunctival scarring has progressed slowly in a medial direction over the subsequent four years but there have been no further episodes of corneal ulceration and visual acuity is normal (6/9 each eye using Sheridan-Gardner test cards).

In addition she has suffered from recurrent loss of toe and finger nails since one year of age. The nails of first and fifth toes of both feet are now missing with replacement of the nailbed by granulation tissue (Fig. 7). A papular rash has been present on the arms and the face for the past year (Fig. 8). The mass on the vocal cords has recurred (Fig. 9) with spread to involve most of the larynx. Increasing stridor has necessitated two further excisions of the abnormal laryngeal tissue and a permanent tracheostomy is contemplated. The deciduous molar and premolar teeth are yellow in appearance due to failure of enamel formation.

Her parents are from the Punjab region of India and are unrelated. She is now five years old, and has a seven year old sister, one halfsister and two half-brothers who are all well.

\section{Laboratory features}

Extensive investigations similar to those of case 1 were unrewarding. Normal adnexal skin structures were present on both clinical and histological examination. At no time were blisters identified on skin or mucous membranes. Pathological findings were identical to case 1: conjunctival, laryngeal and nailbed biopsies revealed subepithelial granulation tissue and ulceration only. Repeated cultures for possible pathogenic organisms were negative.

\section{Discussion}

Clinical examination, histology and biochemical investigation have excluded the presence of epidermolysis bullosa, ectodermal dysplasia, cicatricial pemphigoid, chronic 


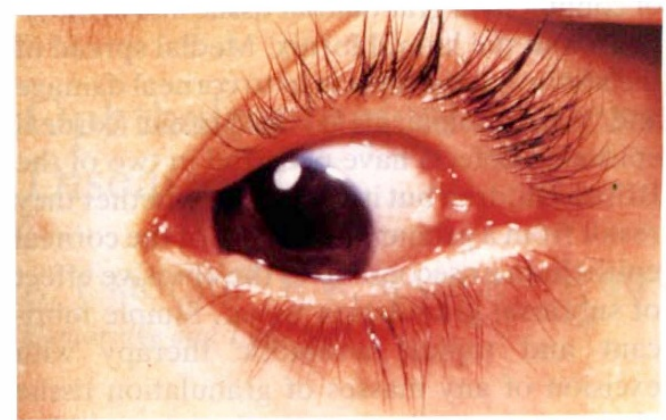

Fig. 6a.

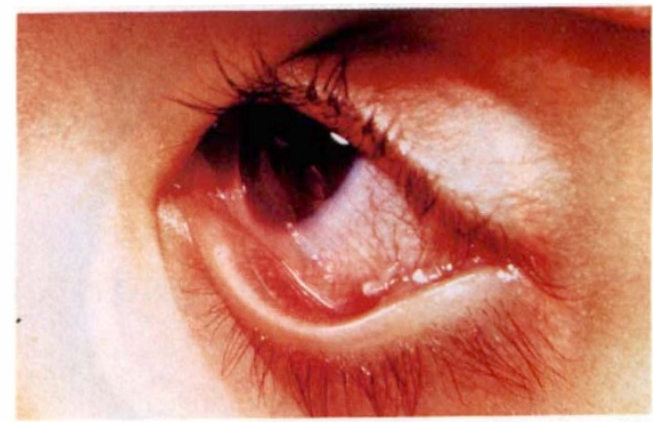

Fig. 6b.

Fig. 6. Case 2 at three years of age. The early ocular changes consist of (a) granulation tissue and (b) symblepharon formation in the lateral fornix.

granulomatous disease of childhood, porphyria, lysosomal storage disease, amyloidosis, lipoproteinosis, immune deficiency and chromosomal abnormality. There has been no evidence of an infective organism on extensive histological and serological investigation or culture. A trial of anti-tuberculous therapy produced no benefit in the older child.

These two children are thought to be suffering from a severe form of a condition previously described on one occasion only, when the name Laryngo-Onycho-Cutaneous syndrome $^{3}$ was used. The journal containing the report ${ }^{3}$ is not available outside Pakistan, and does not appear on international listings. Twenty-two children from 12 families were studied. The children documented in that series were not examined by an ophthalmologist, and no ocular abnormality is mentioned in the paper. All suffered a hoarse voice, but no laryngeal abnormality was seen

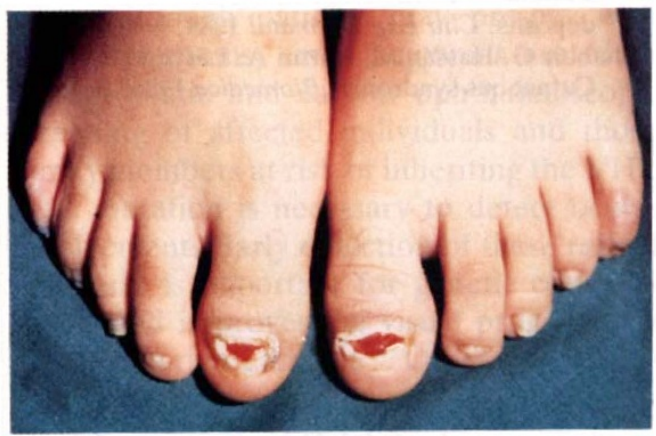

Fig. 7. Case 2 has lost the first and fifth nails of both feet and there is granulation tissue in the nailbed. Repeated fungal cultures have been negative. during a single examination under anaesthetic of each child at the time of diagnosis. Unfortunately, investigation of the children was hampered by a lack of medical resources and support services.

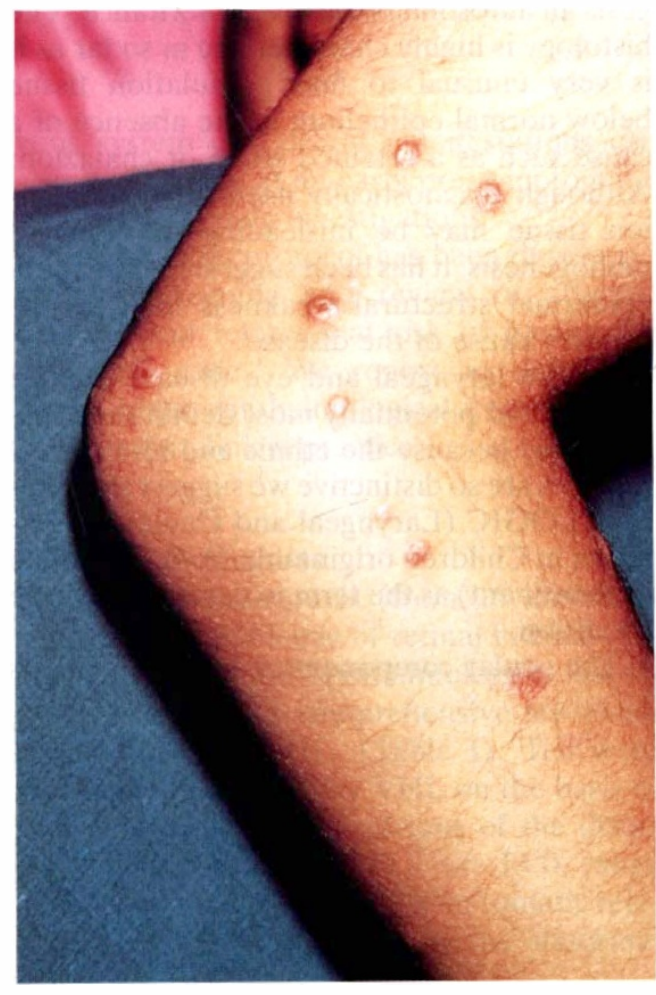

Fig. 8. Case 2 at five years of age. A papular skin eruption has developed which is largely confined to the arms and face. Pruritus leads to excoriation of the papules. 


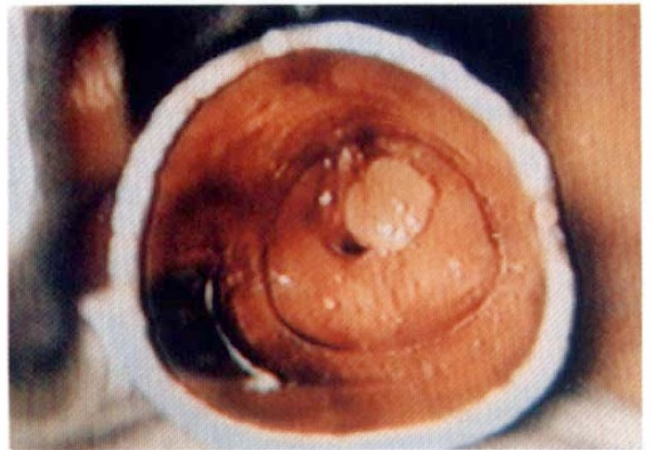

Fig. 9. Larynx of case 2 at three years of age. The mass of submucosal granulation tissue has recurred on the vocal cord.

Children with this syndrome are from Muslim families originating in the Punjab region of northwest India and Pakistan. There is a high rate of consanguinity amongst the parents which, with the frequent occurrence of two affected children in one family, suggests an autosomal recessive inheritance. The histology is highly characteristic in so far as it is very unusual to find granulation tissue below normal epithelium in the absence of a cause such as a retained stitch or chalazion. Although diagnostically useful the granulation tissue may be misleading in terms of pathogenesis: it has been suggested that a subepidermal structural weakness may be the primary cause of the disease.

As the laryngeal and eye disease are the earliest and potentially most debilitating features and because the ethnic and histological features are so distinctive we suggest the acronym LOGIC (Laryngeal and Ocular Granulation in Children originating from the Indian subcontinent) as the term to describe this rare condition.

The ocular component of LOGIC consists of conjunctival granulation tissue and symblepharon at the lateral fornix. Medial spread of the abnormalities may lead to corneal damage and visual impairment. Recurrent corneal epithelial defects have occurred in two of the British children but it is not clear whether they result from a primary instability of the corneal epithelium or secondary to the abrasive effect of subtarsal granulation tissue. Simple lubricant and topical antibiotic therapy with excision of any masses of granulation tissue obviously contributing to the erosion has allowed healing with minimal or no corneal scar.

It is apparent that the pattern and severity of organ involvement in LOGIC varies between children. There is no known pathogenesis for LOGIC, and attempts at treatment with systemic steroids, dapsone and local excision have been without benefit. Further study is urgently required to understand the genetic pattern, pathogenesis and prognosis.

We wish to acknowledge the assistance of the following: Professor G. Shabbir, Professor R. MacKie, Dr W. N. Morley, Dr A. Patrick, Dr A. A. M. Gibson, Dr A. C. E. McCartney, Dr M. J. Tidman.

Key words: Laryngo- Onycho- Cutaneous syndrome, Granulation tissue, LOGIC syndrome, Conjunctival Disease, Laryngeal disease.

\section{References}

${ }^{1}$ McDonnell PJ, Schofield OMV, Spalton DJ, Mayou BJ, Eady RAJ: The eye in Dystrophic Epidermolysis bullosa: clinical and immunopathological findings. Eye 1989, 3; 79-83.

${ }^{2}$ Wojnarowska F, Marsden RA, Bhogal B, Black MM; Childhood cicatricial pemphigoid with linear IgA deposits. Clin Exp Dermatol 1984, 9: 407-15.

${ }^{3}$ Shabbir G, Hassan M, Kazmi A: Laryngo- Onycho. Cutaneous syndrome. Biomedica 1986, 2: 15-25. 